\title{
Scalar gravitation: A laboratory for numerical relativity. II. Disks
}

\author{
Stuart L. Shapiro and Saul A. Teukolsky \\ Center for Radiophysics and Space Research and Departments of Astronomy and Physics, Cornell University, Ithaca, New York 14853
}

(Received 27 August 1993)

\begin{abstract}
While not a correct physical theory, relativistic scalar gravitation provides a simple test site for developing many of the tools of numerical relativity. Using this theory, we have built a mean-field particle simulation scheme to study the dynamical behavior of collisionless disks. Disks are one-dimensional matter sources of two-dimensional gravitational fields. One-dimensional disk sources can be evolved without excessive computational resources and yet they are able to generate nonspherical gravitational waves. We find that we are able to calculate smooth and accurate wave forms from time-varying disks, despite the stochastic representation of the matter source terms caused by sampling with a finite number of particles. A similar scheme should provide accurate wave forms in general relativity, provided sufficient computer resources are used.
\end{abstract}

PACS number(s): 04.25.Dm, 02.70.Bf, 03.50.Kk, 04.30.Db

\section{INTRODUCTION}

A number of codes for solving Einstein's equations of general relativity for nonspherical systems have been developed. By and large, they are restricted to simple systems with axisymmetry (see, e.g., Ref. [1] and references therein). Despite the large amount of effort invested in developing these codes, they cannot track the evolution of general configurations to arbitrarily late times with high accuracy. The technical complications [2] that beset this enterprise make developing and testing new algorithms for numerical relativity very difficult: the human and computational resources required to simulate even the simplest dynamical problems can be considerable.

We have previously demonstrated how a simple theory of relativistic gravitation can be used as a test site for developing many of the tools of numerical relativity [2]. (This paper will referred to as paper I, and numbered equations from that paper will be labeled with I here.) The theory we consider describes gravitation by a nonlinear scalar gravitational field in special relativity and is presented in Exercise 7.1 of Ref. [3]. Of course, this theory disagrees violently with experiment, giving incorrect predictions for the three classical tests of general relativity. Our purpose, however, is to use the theory to develop numerical tools that can then be applied to solve problems in general relativity. In addition, by studying scalar gravitation we can examine some of the generic features of a nonlinear dynamical theory of gravitation in a simple setting, such as gravitational collapse and wave production.

In paper I we confined our attention to spherical systems, exploiting the fact that scalar gravitational waves can be generated even in spherical symmetry. Here we extend our treatment to nonspherical systems with axial symmetry. Techniques for nonspherical systems are required to treat radiating systems in general relativity, where there are no gravitational waves in spherical symmetry. In order to develop tools to solve the field equa- tions, we need to focus our effort on solving those equations, and not on the accompanying matter equations. We thus employ the simplest matter sources that can generate waves in two spatial dimensions. One simple source is an infinitesimally thin disk of collisionless particles in the equatorial plane. Collisionless matter is described by a system of ordinary differential equations. These equations are much easier to solve than, for example, the partial differential equations that describe hydrodynamic matter.

As in paper I, we imagine that the matter consists of a large number of particles of infinitesimal rest mass. In this limit the matter can be characterized by a smooth phase-space distribution function satisfying the Vlasov equation. By integrating the equations of motion in the background scalar gravitational field for a sample of particles from the distribution, we solve the Vlasov equation by a mean-field particle simulation scheme. Since we restrict the particle motion to a plane, we reduce the dynamical degrees of freedom that characterize the particle distribution. Not only are there fewer equations per particle, but also the number of particles required to adequately sample the phase-space distribution is considerably reduced. In fact, the number of degrees of freedom for a disk is the same as for a sphere, even though the gravitational field is two dimensional.

The challenge of handling a disk of particles with a finite-difference code is that the density source term for the field equation is infinite. This produces a discontinuity in the gradient of the gravitational potential. Without special treatment, such a discontinuity would be a disaster for a finite-difference method. We show that a straightforward implementation of a jump condition across the disk can handle discontinuities accurately.

A disk of collisionless particles can also generate gravitational waves in general relativity. Even though the field equations in general relativity are considerably more complicated than in the scalar theory treated here, it should be possible to use the same techniques to handle discontinuities in the matter source terms. Thus the 
demonstration in this paper, that disk systems can be treated with reasonable accuracy, has important implications for evolving disk systems in general relativity. And once we can evolve disk systems accurately in general relativity, we can evolve any axisymmetric source. The reason is that the field equations for disk sources are essentially the same as the field equations for more general axisymmetric sources.

\section{BASIC EQUATIONS}

The basis equations for the scalar gravitation theory discussed here are presented in Sec. II of paper I. The key dynamical equation for the gravitational field $\Phi$ is

$$
\square \Phi=4 \pi G e^{\Phi} \rho .
$$

Here $\rho$ is the comoving matter density. The scalar field does not modify the background space-time geometry, which is always Minkowskian. We adopt spherical polar coordinates and set $x=\cos \theta$. In axisymmetry, Eq. (2.1) becomes

$$
\begin{aligned}
\Phi_{, t t} & =\nabla^{2} \Phi-4 \pi G e^{\Phi} \rho \\
& =\frac{1}{r^{2}}\left(r^{2} \Phi_{, r}\right)_{, r}+\frac{1}{r^{2}}\left[\left(1-x^{2}\right) \Phi_{, x}\right]_{, x}-4 \pi G e^{\Phi} \rho .
\end{aligned}
$$

We will generally set the speed of light $c=1$, but will display the gravitational coupling constant (Newton's constant) $G$ explicitly.

A disk in the equatorial plane is described by a surface density:

$$
\sigma(r)=\int \rho d z=\int \rho r d x .
$$

Integrating Eq. (2.2) across the equatorial plane yields the jump condition

$$
\left.\frac{1}{r} \Phi_{, x}\right|_{+}-\left.\frac{1}{r} \Phi_{, x}\right|_{-}=4 \pi G e^{\Phi} \sigma,
$$

where + refers to $x=0^{+}$and - to $x=0^{-}$. Because $\Phi$ is symmetric across the equator, its derivative is antisymmetric, and so

$$
\left.\frac{1}{r} \Phi_{, x}\right|_{+}=-\left.\frac{1}{r} \Phi_{, x}\right|_{-}=2 \pi G e^{\Phi} \sigma .
$$

Because the particles in the disk are confined to the equatorial plane, the four-velocity component $u_{\theta}=0$. Thus the particle equations of motion are identical to Eqs. (I.4.2)-(I.4.4) for spherical symmetry.

The equation of energy conservation (I.2.19) remains valid, only now $\nabla \phi$ has both $r$ and $x$ components.

\section{RADIATION}

In the weak-field slow-motion limit, the radiation field can be obtained by a multipole expansion. The leadingorder contribution is given by Eq. (I.3.7):

$$
\begin{gathered}
\Phi(t, \mathbf{r})=-\frac{G}{r} \int d^{3} r^{\prime}\left[\rho_{0}\left[\Phi-\frac{1}{2} v^{2}\right]+r^{\prime} \cos \psi \rho_{0, t}\right. \\
\left.+\frac{1}{2} r^{\prime 2} \cos ^{2} \psi \rho_{0, t t}\right]_{t-r},
\end{gathered}
$$

where

$$
\cos \psi=\frac{\mathbf{r} \cdot \mathbf{r}^{\prime}}{r r^{\prime}}
$$

Writing

$\mathbf{r}=r x \mathbf{e}_{z}+r\left(1-x^{2}\right)^{1 / 2} \cos \phi \mathbf{e}_{x}+r\left(1-x^{2}\right)^{1 / 2} \sin \phi \mathbf{e}_{y}$

and a similar equation for $\mathbf{r}^{\prime}$ gives

$$
\begin{aligned}
\cos \psi=x x^{\prime}+ & \left(1-x^{2}\right)^{1 / 2}\left(1-x^{\prime 2}\right)^{1 / 2} \\
& \times\left(\cos \phi \cos \phi^{\prime}+\sin \phi \sin \phi^{\prime}\right) .
\end{aligned}
$$

Using Eq. (3.4) in (3.1), and assuming equatorial and axial symmetry gives

$$
\begin{aligned}
\Phi(t, \mathbf{r})=-\frac{G}{r} \int d^{3} r^{\prime}\left[\rho_{0}\left[\Phi-\frac{1}{2} v^{2}\right]\right. \\
\left.+\frac{1}{4} r^{\prime 2} \rho_{0, t t}\left\{x^{\prime 2}\left(3 x^{2}-1\right)+\left(1-x^{2}\right)\right\}\right]_{t-r}
\end{aligned}
$$

For a disk source, $x^{\prime}=0$, and so

$$
\begin{aligned}
\Phi(t, \mathbf{r})=-\frac{G}{r} \int d^{3} r^{\prime} & {\left[\rho_{0}\left[\Phi-\frac{1}{2} v^{2}\right]\right.} \\
& \left.+\frac{1}{4} r^{\prime 2} \rho_{0, t t}\left(1-x^{2}\right)\right]_{t-r}(\text { disk })
\end{aligned}
$$

This equation is the analogue of the "quadrupole formula" in general relativity. Dimensional estimates of the wave amplitude and emission rates are given in Eqs. (I.3.9)-(I.3.12).

\section{NUMERICAL METHOD}

The finite difference scheme we adopt to integrate Eq. (2.2) is an extension of the one employed in paper I. We again employ an explicit leapfrog method, allowing for a variable time step. We introduce the variable

$$
\lambda \equiv \Phi_{, t}
$$

to split Eq. (2.2) into two first-order equations:

$$
\begin{aligned}
& \mathcal{T}[\Phi]=\lambda, \\
& \mathcal{T}[\lambda]=\mathcal{R}[\Phi]+\mathscr{F}[\Phi],
\end{aligned}
$$

where 


$$
\begin{aligned}
& \mathcal{T}[Y] \equiv Y_{, t}, \\
& \mathcal{R}[\Phi] \equiv 6\left[r^{3} \Phi_{, r^{2}}\right]_{, r^{3}}, \\
& \mathcal{F}[\Phi] \equiv \frac{2}{r^{2}}\left(1-3 x^{2}\right)\left[\left(x-x^{3}\right) \Phi_{, x^{2}}\right]_{,\left(x-x^{3}\right)} .
\end{aligned}
$$

Here $Y$ denotes either $\Phi$ or $\lambda$. The Laplacian in the field equation has been written in the form shown in Eq. (4.3) to ensure regularity in the finite-difference operator near the origin and the axis (cf. Appendix of Ref. [4] for the radial term). The source term in Eq. (2.2) is incorporated through the boundary conditions discussed below.

To finite difference the equations, introduce a radial grid $\left\{r_{i}\right\}, i=1,2, \ldots, i_{\max }$, extending from $r=0$ up to the outer boundary $r=r_{\max }$, and an angular grid $\left\{x_{k}\right\}$, $k=1,2, \ldots, k_{\max }$, extending from the axis at $x=-1$ up to the equator at $x=0$. Here we have incorporated the equatorial symmetry of the configuration. The prescription for laying down the radial grid is given in paper I. The angular grid is chosen to be uniform. We take the fields $\Phi$ and $\lambda$ to be defined at the midpoint of zones. The finite difference form of the operators in equation (4.3) is then

$$
\begin{aligned}
\mathcal{T}_{i+1 / 2, k+1 / 2}^{n}[Y]= & \frac{\Delta t_{n-1}}{\Delta t_{n}+\Delta t_{n-1}} \frac{Y_{i+1 / 2, k+1 / 2}^{n+1}-Y_{i+1 / 2, k+1 / 2}^{n}}{\Delta t_{n}}+\frac{\Delta t_{n}}{\Delta t_{n}+\Delta t_{n-1}} \frac{Y_{i+1 / 2, k+1 / 2}^{n}-Y_{i+1 / 2, k+1 / 2}^{n-1}}{\Delta t_{n-1}}, \\
\mathcal{R}_{i+1 / 2, k+1 / 2}^{n}[\Phi]= & \frac{6}{r_{i+1}^{3}-r_{i}^{3}}\left[r_{i+1}^{3} \frac{\Phi_{i+3 / 2, k+1 / 2}^{n}-\Phi_{i+1 / 2, k+1 / 2}^{n}}{r_{i+3 / 2}^{2}-r_{i+1 / 2}^{2}}-r_{i}^{3} \frac{\Phi_{i+1 / 2, k+1 / 2}^{n}-\Phi_{i-1 / 2, k+1 / 2}^{n}}{r_{i+1 / 2}^{2}-r_{i-1 / 2}^{2}}\right], \\
\mathcal{F}_{i+1 / 2, k+1 / 2}^{n}[\boldsymbol{\Phi}]= & \frac{2}{r_{i+1 / 2}^{2}} \frac{1-3 x_{k+1 / 2}^{2}}{\left(x-x^{3}\right)_{k+1}-\left(x-x^{3}\right)_{k}} \\
& \times\left[\left(x-x^{3}\right)_{k+1} \frac{\Phi_{i+1 / 2, k+3 / 2}^{n}-\Phi_{i+1 / 2, k+1 / 2}^{n}}{x_{k+3 / 2}^{2}-x_{k+1 / 2}^{2}}-\left(x-x^{3}\right)_{k} \frac{\Phi_{i+1 / 2, k+1 / 2}^{2}-\Phi_{i+1 / 2, k-1 / 2}^{n}}{x_{k+1 / 2}^{2}-x_{k-1 / 2}^{2}}\right],
\end{aligned}
$$

where $\Delta t_{n}=t_{n+1}-t_{n}$. These operators are second-order accurate in both space and time.

We impose an outgoing wave boundary condition at $r=r_{\max }$, as described by Eqs. (I.5.5)-(I.5.7). We do not need to explicitly impose a boundary condition at the origin: when evaluated for $i=1$, the term containing $\Phi_{1 / 2, k+1 / 2}$ in Eq. (4.4) is multiplied by $\left(r_{1}\right)^{2}=0$. Similarly, we do not need to impose a boundary condition on the axis, since when evaluated for $k=1$, the term containing $\Phi_{i+1 / 2,1 / 2}$ in Eq. (4.4) is multiplied by $\left(x-x^{3}\right)_{1}=0$. At the equator, we impose the disk jump condition (2.5):

$\left(x-x^{3}\right) \Phi_{, x^{2}}=\frac{1}{2}\left(1-x^{2}\right) \Phi_{, x}=-\pi G r e^{\Phi_{\sigma}} \quad\left(x=0^{-}\right)$.

In finite-difference form, we make the replacement

$$
\begin{array}{r}
\left(x-x^{3}\right)_{k+1} \frac{\Phi_{i+1 / 2, k+3 / 2}^{n}-\Phi_{i+1 / 2, k+1 / 2}^{n}}{x_{k+3 / 2}^{2}-x_{k+1 / 2}^{2}} \\
=-\pi G r_{i+1 / 2} \exp \left(\Phi_{i+1 / 2, k+1}\right) \sigma_{i+1 / 2} \\
\quad\left(k=k_{\max }-1\right)
\end{array}
$$

in Eq. (4.4). We evaluate $\Phi_{i+1 / 2, k+1}$ appearing on the right-hand side of Eq. (4.6) by extrapolation.

For a uniform Cartesian grid, the Courant stability condition for the above leapfrog scheme would be

$$
\Delta t<\frac{1}{2} \Delta x\left[1+\left(\frac{\Delta x}{\Delta y}\right]^{2}\right]^{-1 / 2},
$$

where $\Delta x=x_{i+1}-x_{i}$ and similarly for $y$. Heuristically adapting this criterion to a variably spaced, spherical polar grid with uneven time steps, we use the stability condition

$\Delta t<\frac{\alpha}{2} \min \left\{\Delta r_{i}\left[1+\left(\frac{\Delta r_{i}}{r_{i} \Delta x_{k}}\right]^{2}\left(1-x_{k}^{2}\right)\right]^{-1 / 2}\right\}$,

with $\alpha<1$. Choosing $\alpha$ smaller decreases the time integration error; typically we find $\alpha=0.5$ is adequate. We find empirically that this criterion yields a stable scheme even when the grid is adjusted on each time step to follow the matter. To prevent the growth of high-frequency instabilities associated with the leapfrog scheme, we add a small Kreiss-Oliger [5] diffusive term to the evolution Eqs. (4.2).

The matter term $\sigma$ appearing in Eq. (4.6) is computed from the particle distribution according to

$$
\sigma=\sum_{A} m_{A}\left(\frac{1}{2 \pi r \Delta r}\right) \frac{1}{\tilde{u}_{A}^{0}} e^{\Phi},
$$

where $m_{A}$ is the rest mass of an individual particle $A$. For equal mass particles,

$$
m_{A}=\frac{M_{0}}{N_{p}}
$$

where $M_{0}$ is the total rest mass and $N_{p}$ is the total number of particles. Using Eq. (I.2.10) and Eq. (2.3), we have

$$
M_{0}=\int \gamma \sigma 2 \pi r d r .
$$


For evaluating the conservation equation (I.2.19), we also need two other matter source terms:

$$
\begin{aligned}
& \int T_{\text {particle }}^{00} d z=\sum m_{A}\left(\frac{1}{2 \pi r \Delta r}\right) \tilde{u}_{A}^{0}, \\
& \int T_{\text {particle }}^{0 r} d z=\sum m_{A}\left[\frac{1}{2 \pi r \Delta r}\right) \tilde{u}_{r}^{A} .
\end{aligned}
$$

To compute smooth radial source profiles, we bin the particles in the disk according to the prescription of
Hockney and Eastwood [6] described in paper I. We choose the radial grid coordinates similar to the scheme in paper I. Since we are dealing with disks, however, in the matter interior we choose the mesh to be uniform in the areal coordinate $r^{2}$ rather than the volume coordinate $r^{3}$.

In the weak-field, slow motion limit, we can use the multipole expansion formula (3.6) as a check on our wave integrations. We cast the equation into a sum over the particle positions and velocities as in paper I. Instead of Eq. (I5.17) we get

$$
r \Phi(t, r, x)=G \sum m_{A}\left[\frac{1}{2}\left[\left(\tilde{u}_{r}^{A}\right)^{2}+\frac{\left(\tilde{u}_{\phi}^{A}\right)^{2}}{r_{A}^{2}}\right]-\Phi-\frac{1}{2}\left(1-x^{2}\right)\left[\left(\tilde{u}_{r}^{A}\right)^{2}+\frac{\left(\tilde{u}_{\phi}^{A}\right)^{2}}{r_{A}^{2}}-r_{A} \Phi_{, r_{A}}\right]\right]_{t-r}
$$

Differentiating Eq. (4.13) with respect to time gives $\lambda$, and one can make substitutions using the equations of motion to simplify the resulting formula. We found instead that it was adequate to take numerical derivatives of Eq. (4.13) to get $\lambda$ for this check.

All the problems we have chosen to analyze start from a moment of time symmetry, so that

$$
\begin{aligned}
& \lambda=0, \\
& \nabla^{2} \Phi=0,
\end{aligned}
$$

subject to the boundary conditions

$$
\begin{aligned}
& \Phi_{, r}=0, \quad r=0, \\
& (r \Phi)_{, r}=0, \quad r \rightarrow \infty, \\
& \Phi_{, \theta}=-\left(1-x^{2}\right)^{1 / 2} \Phi_{, x} \rightarrow 0, \quad x \rightarrow-1, \\
& -\frac{1}{r} \Phi_{, x}=2 \pi G e^{\Phi} \sigma, \quad x \rightarrow 0^{-},
\end{aligned}
$$

Equations (4.14)-(4.18) are solved by iteration starting with an initial guess for $\Phi$ on the right-hand side of (4.18). Finite differencing using the operators $\mathcal{R}$ and $\mathcal{F}$ in Eq. (4.4) then gives a sparse system of linear equations that are iterated to convergence.

For the matter, we choose a random sample of particles to represent the given initial density and velocity profiles. For sufficient resources, this scheme yields initial data of arbitrary accuracy even for strong fields and high particle velocities.

\section{ANALYTIC TESTS}

\section{A. Oscillating Newtonian disks}

In general, satisfying the conservation equation (I.2.19) provides a self-consistent check on a numerical integration. However, as in the spherical case, there is also a complete analytic solution that furnishes a good test for weak fields and slow velocities. The solution describes an oscillating homogeneous spheroid in Newtonian gravita- tion in the disk limit (eccentricity $e \rightarrow 1$ ). The surface density of such a disk of mass $M$ and radius $R$ is

$$
\sigma(r)=\frac{3 M}{2 \pi R^{2}}\left[1-\frac{r^{2}}{R^{2}}\right]^{1 / 2} \text {. }
$$

Start with the equation of motion for the semimajor axis $R$ of an oblate homogeneous spheroid (e.g., Eq. 5.6 of Ref. [4]). Take the limit $e \rightarrow 1$ and find

$$
\ddot{R}=-\frac{3 \pi}{4} \frac{G M}{R^{2}}+\frac{h^{2}}{R^{3}},
$$

where $h$ is the conserved angular momentum per unit mass of a particle at the surface. Since the motion is homologous, the radius of each particle satisfies a similar equation. Choose $h$ to be a fraction $\xi$ of the equilibrium angular momentum $h_{0}=\left(3 \pi G M R_{0} / 4\right)^{1 / 2}$. Set

$$
R=R_{0} X(t) .
$$

Then the radius $r$ of each particle satisfies

$$
r=r_{0} X(t)
$$

where $r_{0}$ is the initial radius. Substituting (5.3) into (5.2) we see that $X$ satisfies the familiar equation of an elliptic orbit for a particle with specific angular momentum $h_{\text {eff }}=\xi\left(M / R_{0}^{3}\right)^{1 / 2}$ around a fixed central mass $M_{\text {eff }}=3 \pi M / 4$. (We discussed a similar model for oscillations of a homogeneous sphere in Appendix C of Ref. [7] and in paper I.) The parametric solution for $X(t)$ is

$$
\begin{aligned}
& X=a(1-e \cos u), \\
& t=\frac{P}{2 \pi}(u-e \sin u)-\frac{P}{2},
\end{aligned}
$$

where we assume $X=1$ and $\dot{X}=0$ at $t=0$. In Eq. (5.5), the semimajor axis, eccentricity, and period are given by 


$$
\begin{aligned}
& a=\frac{1}{2-\xi^{2}}, \\
& e=1-\xi^{2}, \\
& P=2 \pi\left(\frac{4 R_{0}^{3}}{3 \pi G M\left(2-\xi^{2}\right)^{3}}\right)^{1 / 2} .
\end{aligned}
$$

The radial and tangential particle velocities are given by

$$
\begin{aligned}
& v_{r}=\frac{\dot{X}}{X} r \\
& v_{\phi}=\xi \frac{r}{X^{2}}\left(\frac{3 \pi G M}{4 R_{0}^{3}}\right)^{1 / 2} .
\end{aligned}
$$

Inserting this analytic solution into Eq. (3.6) and differentiating with respect to time gives the wave amplitude

$$
r \lambda=-\frac{3 \pi}{20} \frac{(G M)^{2}}{R_{0}}\left(5+\cos ^{2} \theta\right)\left[\frac{\dot{X}}{X^{2}}\right]_{t-r} \quad \text { (wave zone) } .
$$

Thus the rate of energy emission is

$$
\begin{aligned}
\frac{d E}{d t} & =\int r^{2} T_{0 r}^{\mathrm{field}} d \Omega \\
& =\frac{321 \pi^{2}}{580} \frac{(G M)^{4}}{R_{0}^{2}}\left[\frac{\dot{X}^{2}}{X^{4}}\right]_{t-r} .
\end{aligned}
$$

\section{B. Kalnajs disks}

When $\xi=1$, the above solution corresponds to a uniformly rotating disk in dynamical equilibrium. It is well known that such a disk is unstable. In axisymmetry, the disk is unstable to the formation of concentric rings. The disk can be stabilized by "heating" the disk, that is, converting some of the ordered rotational energy into random "thermal" motion. Kalnajs [8] has given an analytic prescription for constructing hot homogeneous disks in equilibrium. They all have the same surface density $\sigma$ and gravitational potential $\Phi$ as the cold disk in Sec. V A, but differ in the amount of random motion. In these models, the particles have an isotropic velocity distribution in a rotating frame that moves with angular velocity $\Omega$ :

$$
\begin{aligned}
& v \cos \chi=v_{\phi}-\Omega r, \\
& v \sin \chi=v_{r} .
\end{aligned}
$$

Here $v$ is the magnitude of the isotropic velocity in the rotating frame and $\chi$ is a random angle about the particle position in the disk plane. The distribution of particle velocities is given by

$$
f(v) v d v=2 \pi K\left[v_{\max }^{2}-v^{2}\right]^{-1 / 2} v d v .
$$

Here

$$
v_{\max }^{2}=\left(\Omega_{0}^{2}-\Omega^{2}\right)\left(R_{0}^{2}-r^{2}\right),
$$

$$
\Omega_{0}=\frac{h_{0}}{R_{0}^{2}}
$$

and $K$ is a normalization constant. Models in this family are parametrized by the ratio $\Omega / \Omega_{0}$. Cold disks have $\Omega=\Omega_{0}$. Hot disks with $\Omega / \Omega_{0}<0.816$ are stable against ring formation.

\section{NUMERICAL RESULTS}

When analyzing our numerical results, we will adopt gravitational units where $G=c=1$.

\section{A. Oscillating Newtonian disks}

As a check, one would first like to simulate the collapse of the oscillating homogeneous disk described in Sec. V A. Unfortunately, such a cold configuration is unstable to ring formation. This is the familiar result for cold equilibrium disks with $\Omega=\Omega_{0}$ and $\xi=1$ discussed above; we find numerically that it also holds for cold oscillating disks. We can still employ this analytic model to check the field solver in our code provided we supply the unperturbed source function $\sigma$ analytically as a function of time. We then allow the code to solve the field equations (4.2) and thereby determine the radiation numerically.

In Fig. 1 we follow the oscillation of a homogeneous disk with initial radius $R_{0} / M_{0}=1000$ and velocity cutdown factor $\xi=0.7$. This choice of radius is sufficiently large that the system remains essentially Newtonian throughout its evolution. We set the outer grid at $r_{\max } / M_{0}=1000$. We use 60 interior radial zones, 180 exterior radial zones, and 16 angular zones.

The emission of gravitational waves is a postNewtonian effect in the system. Figure 1 shows the wave amplitude $\lambda$ measured at a fixed exterior radius $r=1000 M_{0}$. The amplitude agrees well with the analytic

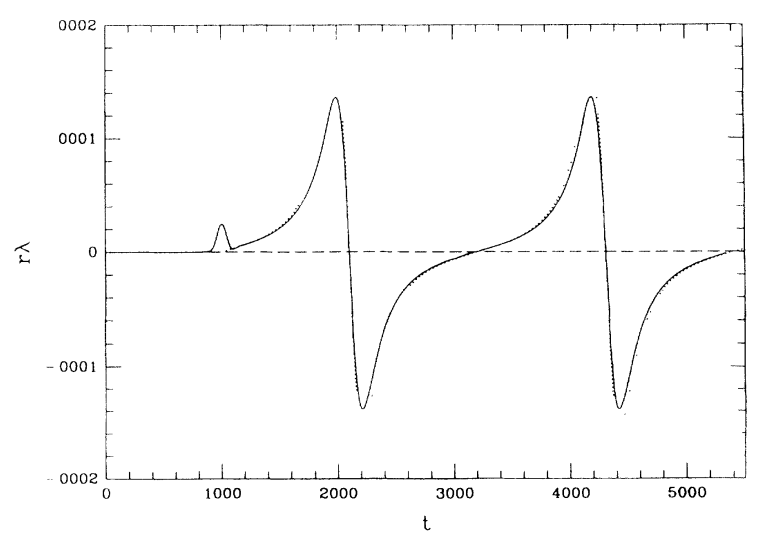

FIG. 1. Wave amplitude $\lambda$ (multiplied by $r$ ) as a function of time as measured at $r=1000 M_{0}$ and $\cos \theta=0.53$ for an oscillating homogeneous Newtonian disk. The solid line shows the numerical result obtained from Eq. (4.2), and the dotted line is the analytic result, Eq. (5.8). The small bump near $t=1000$ is an artifact of slightly imprecise numerical initial data. $r \lambda$ is dimensionless, while $t$ is in units of $M_{0}$. 
amplitude (5.8), which is also shown in the plot. There are two causes of the residual discrepancy. The first is due to post-Newtonian corrections to the analytic amplitude, which are maximal at the bounce radius, $r \approx 30 M$. We have verified that the agreement with the analytic result is better when the system collapses from a larger radius and is therefore more Newtonian. The second contribution to the discrepancy occurs because the wavelength is approximately $2000 \mathrm{M}$, while the outermost grid point is only at $1000 \mathrm{M}$. The field at this point still contains small contributions from non- $(1 / r)$ terms. In Ref. [9] we present a more elaborate technique for accurately extracting the true $1 / r$ piece of the field even at radii less than a wavelength. The overall agreement between the analytic and numerical results here gives some confidence in the reliability of the numerical implementation of the jump conditions and the solution of the field equations.

\section{B. Oscillating Kalnajs disks}

To test the particle simulation aspects of our code, we set up stable equilibrium Kalnajs disks as described in Sec. V B. Our code successfully holds these in equilibrium for several rotation periods.

We next study the production of scalar gravitational radiation from the oscillation of a hot nonequilibrium disk. We set up a Kalnajs disk with $R_{0} / M_{0}=100$ and $\Omega / \Omega_{0}=0.7$. We then induce collapse by reducing all particle velocity components by a factor $\xi=0.7$. Figure 2 shows the wave amplitude observed at radius $r / M_{0}=1000$. For comparison, we plot the amplitude calculated from the multipole formula (4.13) using the particle positions and velocities. The good agreement over several oscillations tests many facets of the meanfield particle simulation scheme, including the field and particle integrators, the particle and grid sampling, and the source binning algorithm.

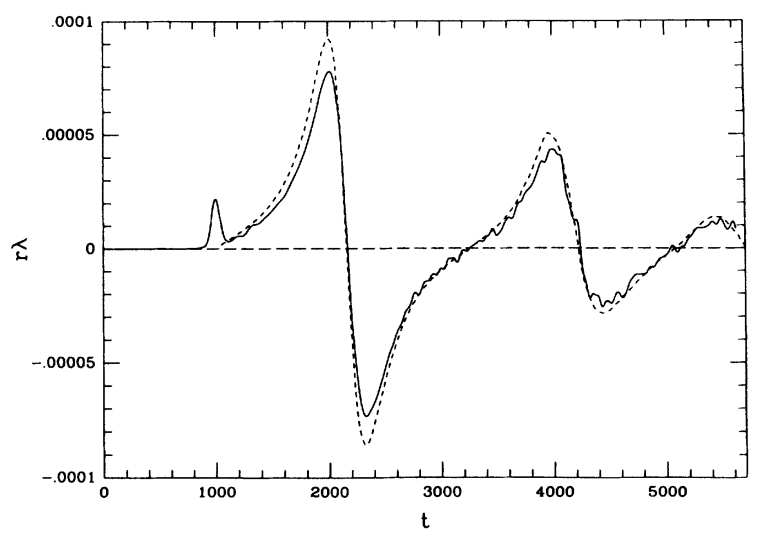

FIG. 2. Wave amplitude $\lambda$ (multiplied by $r$ ) as a function of time as measured at $r=1000 M_{0} \cos \theta=0.53$ for an oscillating Kalnajs disk. The solid line shows the numerical result obtained from Eq. (4.2), while the dashed line is the result from the multipole formula (4.13) numerically differentiated with respect to time.

\section{Oscillating relativistic disk}

To explore scalar gravitation for disks in the nonlinear gravitational field regime, we consider the oscillation of relativistic disks. Take the disks to have an initial density profile given by

$$
\nabla^{2} \Phi=4 \pi G e^{\Phi} \rho \equiv 4 \pi G \rho_{N} .
$$

Here $\rho_{N}$ is the value of the constant density inside a Newtonian disk of the same radius. Integrating $\rho$ through the disk yields

$$
\sigma=\sigma_{N} e^{-\Phi_{N}},
$$

where $\sigma_{N}$ is the Newtonian surface density

$$
\sigma_{N}(r)=\frac{3 M_{N}}{2 \pi R_{0}^{2}}\left[1-\frac{r^{2}}{R_{0}^{2}}\right]^{1 / 2}
$$

(cf. Eq. 5.1). This choice of density gives an analytic solution for $\Phi$, namely, the Newtonian value

$$
\Phi=\Phi_{N}=-\frac{3 \pi M_{N}}{4 R_{0}}\left[1-\frac{r^{2}}{2 R_{0}^{2}}\right] \quad \text { (inside) . }
$$

Choose the initial radial velocity to be zero, and the tangential velocity to be

$$
\tilde{u}_{\phi}^{2}=\xi^{2} r^{3} e^{2 \Phi} \Phi_{, r}
$$

If the cutdown factor $\xi$ is set equal to one, then the cluster is in equilibrium with each particle moving in a circular orbit (cf. Eq. I.4.2). The rest mass is given by Eq. (4.11).

Evolving such a cold cluster (no velocity dispersion) unfortunately leads to the generation of rings, as in the Newtonian case. For example, Fig. 3 shows the collapse
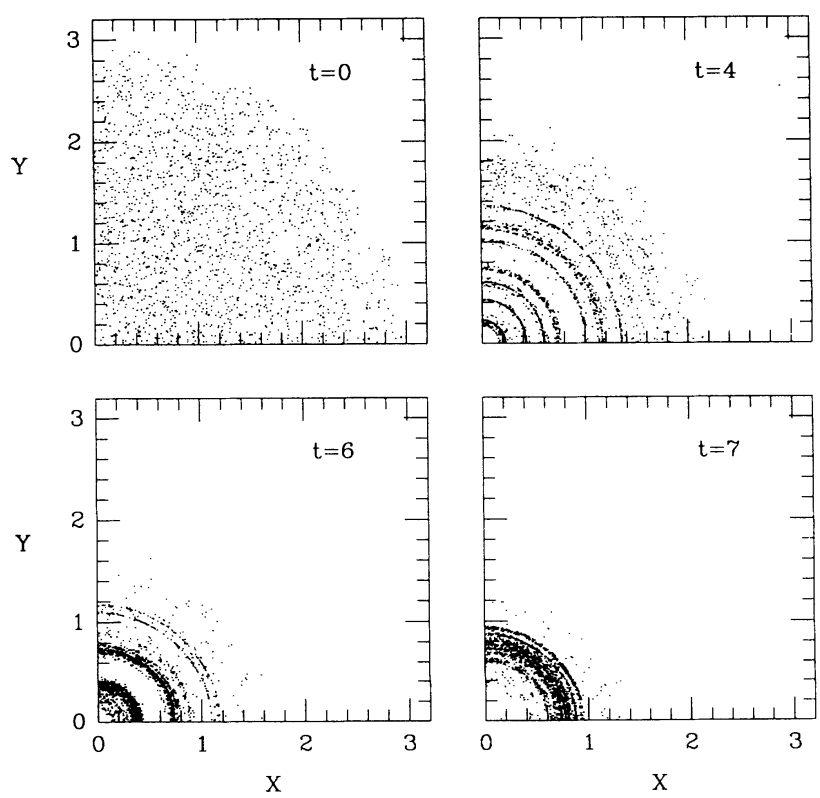

FIG. 3. Snapshots of the particle positions for the evolution of a cold relativistic disk. Particle coordinates and time are in units of $\boldsymbol{M}_{0}$. 


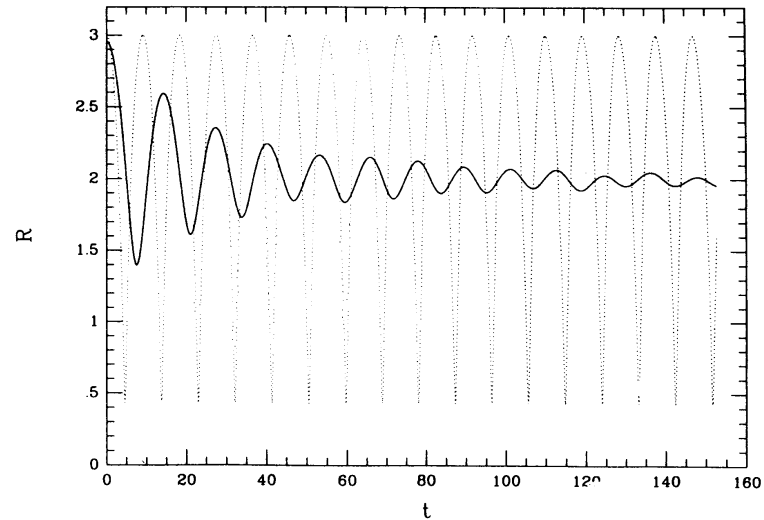

FIG. 4. Evolution of the mean radius as a function of time for an oscillating relativistic Kalnajs disk with initial radius $R_{0} / M_{0}=3, \Omega / \Omega_{0}=0.9$, and $\xi=0.5$. The solid line shows the numerical result obtained from Eq. (6.6). The dotted line is the analytic result, Eq. (5.3), for a cold Newtonian homogeneous disk with the same initial radius and value of $\xi$. Both $R$ and $t$ are in units of $M_{0}$.

of a relativistic disk with $R_{0} / M_{0}=3$ and $\xi=0.5$. Since the disk is highly relativistic, the three masses in scalar gravitation theory defined in Eqs. (I.2.10), (I.2.22), and (I.2.24) are not equal, $M=0.81 M_{0}$ and $M_{C}=0.65 M_{0}$. The unstable growth of rings is evident in the figure. Evolving such a configuration numerically is difficult because of the increasingly singular nature of the particle distribution.

To prevent the formation of rings, we again have to endow the particles with random motion in the plane of the disk. We do this by adopting the Kalnajs recipe of Sec.
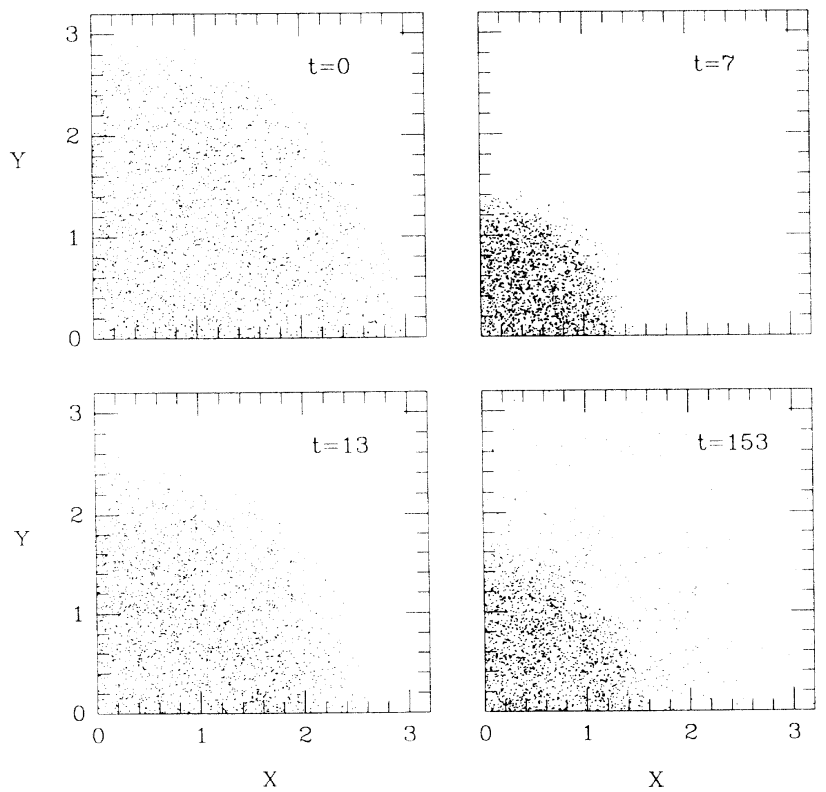

FIG. 5. Snapshots of the particle positions for the evolution of the disk described in Fig. 4. The last snapshot shows the particle configuration at late times when it has almost achieved virial equilibrium.
VB and then self-consistently solving the initial-value equations (4.14)-(4.18). We consider a disk with $R_{0} / M_{0}=3, \Omega / \Omega_{0}=0.9$, and $\xi=0.5$. The masses for this choice of parameters are $M=0.82 M_{0}$ and $M_{C}=0.65 M_{0}$. We run this case with 16000 particles, 60 interior radial zones, 180 exterior radial zones, 16 angular zones, and an outer boundary at $r / M_{0}=50$. Integrating for 45000 timesteps takes approximately 16 hours on a Sun Sparcstation 10. A run with similar resources in general

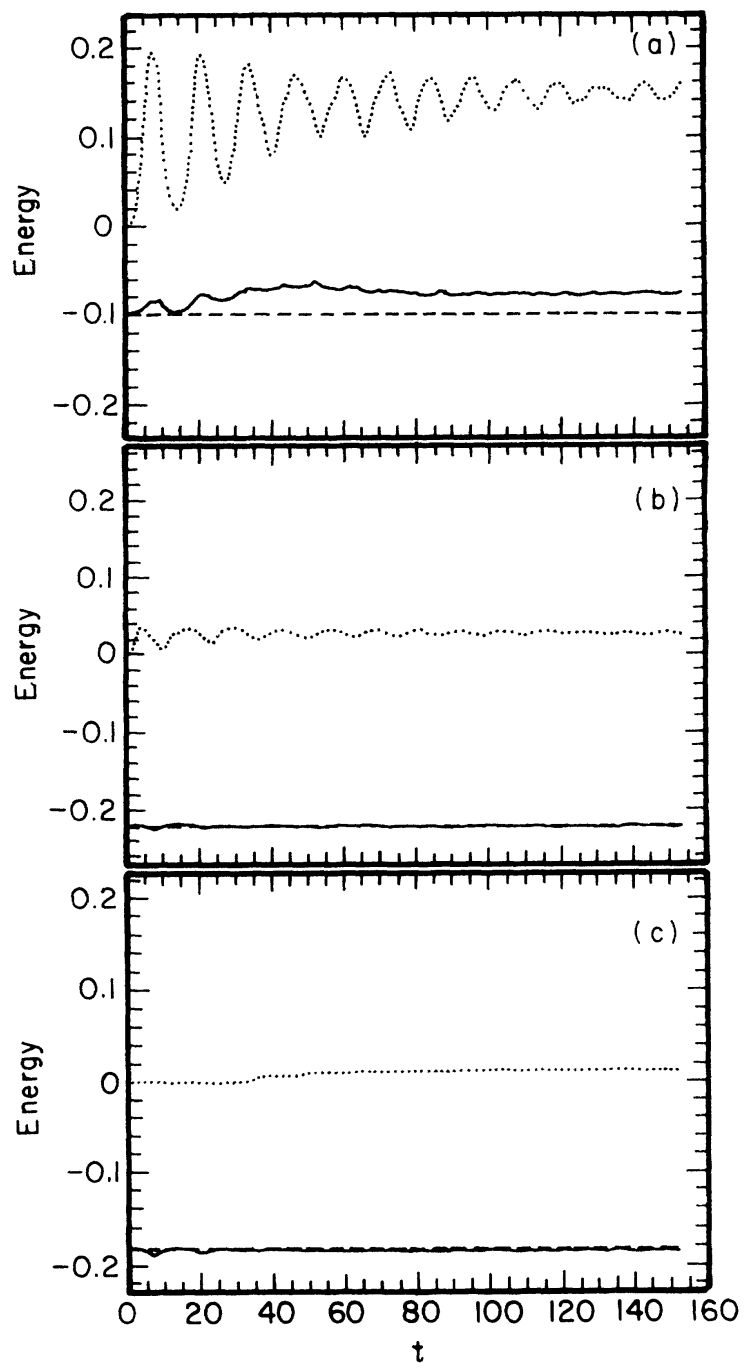

FIG. 6. Energy conservation at three selected radii as a function of time for the evolution described in Fig. 4. The solid line shows the left-hand side of Eq. (I.2.19) (volume integral plus integrated flux), the dotted line shows the second term alone (integrated flux), and the dashed line shows the right-hand side (volume integral at $t=0$ ). The radii are (a) $r=1.4 M_{0}$, (b) $r=2.4 M_{0}$, and (c) $r=25 M_{0}$. Energy and time are in units of $M_{0}$. The degree to which the solid and dashed lines coincide compared with the magnitude of the dotted line is a measure of the code's ability to conserve energy. 


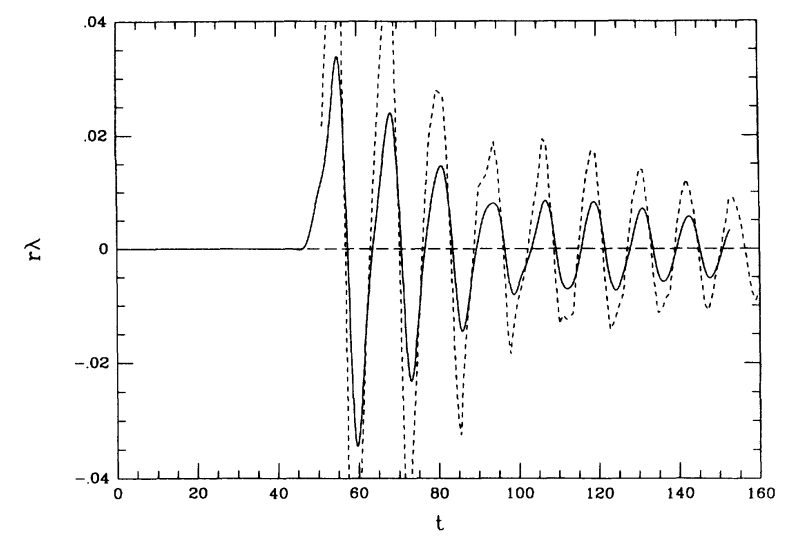

FIG. 7. Wave amplitude $\lambda$ (multiplied by $r$ ) as a function of time as measured at $r=50 M_{0}$ and $\cos \theta=0.53$ for the evolution shown in Fig. 4. Labeling is the same as in Fig. 1.

relativity would take considerably longer.

Figure 4 shows the evolution of the mean radius

$$
R_{\mathrm{rms}} \equiv\left(\frac{5}{2} \frac{1}{N_{p}} \Sigma r_{A}^{2}\right)^{1 / 2}
$$

The factor of $\frac{5}{2}$ has been chosen so that the $R_{\text {rms }}$ will be exactly equal to the radius of a uniform density Newtonian disk represented by a homogeneous particle distribution. The cluster undergoes nonhomologous damped oscillations as violent relaxation drives the system to virial equilibrium. We have checked that the relativistic virial relation (I.2.21) is approached at late times. Figure 5 shows snapshots of the particle positions at selected times. Note that even though $\Omega / \Omega_{0}>0.816$, no rings form in this relativistic dynamical case.

Since there is no analytic solution for this case, the only check we have is provided by the energy conservation equation (I.2.19). We show how well the code conserves energy in Fig. 6. We evaluate the conservation equation as a function of time at three fixed radii. The first radius is inside the matter at all times; the second is alternately inside and outside the matter as the surface oscillates back and forth; and the third radius is always in the vacuum exterior. In the first two cases the integrated flux term (second integral in Eq. I.2.19) is large, and ener- gy conservation involves the small difference of large terms. Consequently, the high degree to which we are able to maintain energy conservation is a nontrivial measure of the accuracy of the code. In the exterior, the flux is small and energy conservation is not a stringent test. However, the wave propagates according to the linear scalar wave equation in the exterior, and the analytic test at small wave amplitude already validated this aspect of the code.

The wave amplitude at fixed radius for this case is plotted as a function of time in Fig. 7. By contrast with the Newtonian example, the wave amplitude in this strong field case is not adequately described by the weak-field multipole expansion formula (4.13). As is evident, the amplitude is slowly damped as the matter oscillations decay.

\section{CONCLUSIONS}

Scalar gravitation is by no means a correct physical theory, but it provides a perfectly fine and simple framework for learning how to do numerical relativity. We have used the theory to test out a mean-field particle simulation scheme for the dynamical evolution of a collisionless disk of matter in a self-consistent gravitational field. A disk is a one-dimensional matter distribution that gives rise to a two-dimensional gravitational field. Such a matter source can be tracked without extravagant computational resources. We find that our numerical method allows one to calculate smooth and accurate gravitational wave forms. Imposing the disk jump conditions on the gravitational field equations can be handled reliably by the numerical scheme. The stochastic representation of the particle distribution is not an inherent obstacle. With sufficient computer resources, a similar disk scheme should be able to calculate reliable gravitational wave amplitudes in general relativity.

\section{ACKNOWLEDGMENTS}

We thank M. Scheel for several useful discussions. This research was supported in part by NSF Grants AST 91-19475 and PHY 90-07834 and NASA Grant NAGW2364 at Cornell University.
[1] C. R. Evans, L. S. Finn, and D. W. Hobill, Frontiers in Numerical Relativity (Cambridge University Press, Cambridge, England, 1989).

[2] S. L. Shapiro and S. A. Teukolsky, Phys. Rev. D 47, 1529 (1993).

[3] C. W. Misner, K. S. Thorne, and J. A. Wheeler, Gravitation (Freeman, San Francisco, 1973).

[4] S. L. Shapiro and S. A. Teukolsky, Astrophys. J. 318, 542 (1987).

[5] H. O. Kreiss and J. Oliger, Methods for the Approximate Solution of Time Dependent Problems, GARP Publication
Series No. 10 (World Meteorological Organization, Geneva, 1973).

[6] R. W. Hockney and J. W. Eastwood, Computer Simulation Using Particles (McGraw-Hill, New York, 1981).

[7] S. L. Shapiro and S. A. Teukolsky, Astrophys. J. 298, 34 (1985).

[8] A. J. Kalnajs, Astrophys. J. 175, 63 (1972); see also J. Binney and S. Tremaine, Galactic Dynamics (Princeton University Press, Princeton, 1987).

[9] M. A. Scheel, S. L. Shapiro, and S. A. Teukolsky, Phys Rev. D 49, 1894 (1994). 

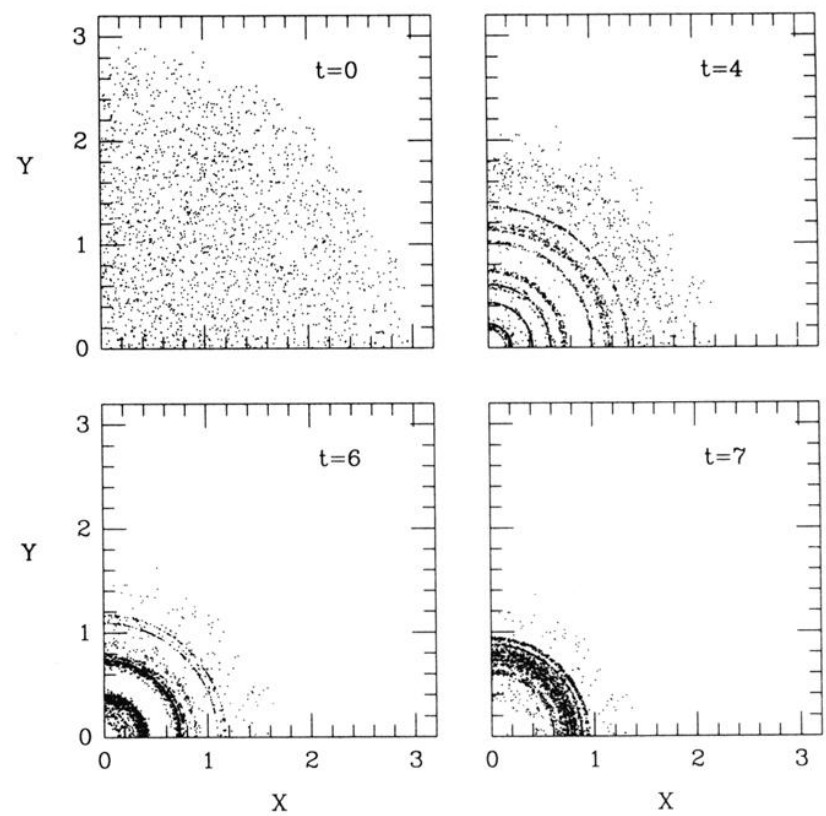

FIG. 3. Snapshots of the particle positions for the evolution of a cold relativistic disk. Particle coordinates and time are in units of $M_{0}$. 

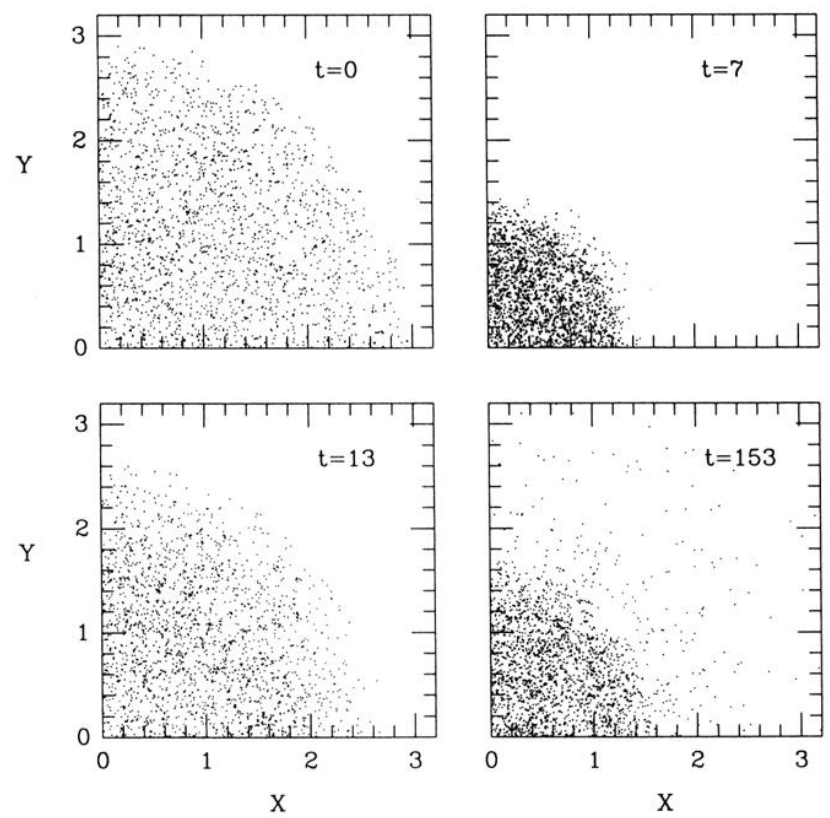

FIG. 5. Snapshots of the particle positions for the evolution of the disk described in Fig. 4. The last snapshot shows the particle configuration at late times when it has almost achieved virial equilibrium. 